Article

\title{
Wavelet Analysis of Microcirculatory Flowmotion Reveals Cardiovascular Regulatory Mechanisms-Data from a Beta-Blocker
}

\author{
Henrique Silva ${ }^{1,2}\left({ }^{\infty}\right.$, Étienne Roux ${ }^{3}$, Alain-Pierre Gadeau ${ }^{3}(\mathbb{D}$ and Luis Monteiro Rodrigues $1, *(\mathbb{1})$ \\ 1 CBiOS (Research Center for Biosciences and Health Technologies), School of Health Sc \& Technologies, \\ Universidade Lusófona, Campo Grande 376, 1749-024 Lisboa, Portugal \\ 2 Pharmacol. Sc Department, Faculty of Pharmacy, Universidade de Lisboa, 1649-004 Lisboa, Portugal \\ 3 Biology of Cardiovascular Diseases, UMR 1034, INSERM, Université de Bordeaux, F-33600 Pessac, France \\ * Correspondence: monteiro.rodrigues@ulusofona.pt
}

Received: 1 May 2020; Accepted: 3 June 2020; Published: 9 June 2020

\begin{abstract}
A variety of animal models exist for the study of cardiovascular function using many approaches from surgically induced ischemia to genetic manipulation. A murine physiological model was recently proposed for the non-invasive study of peripheral circulation and was strengthened by the wavelet transform analysis (WA) of laser Doppler flowmetry (LDF) signals. WA allows the extraction of cardiac, respiratory, sympathetic, endothelial, and myogenic components from the raw LDF signal. The present study was designed to evaluate the discernment capacity of the model through an analysis of the short-term effects of the well-known hypotensive cardiovascular drug, atenolol. Six male C57/BL6 mice (16 weeks old) were included in the study, with each animal serving as its own control. Following anesthesia with ketamine-xylazine, skin perfusions were continuously assessed in both hindlimbs by LDF during baseline and after two sequential atenolol administrations ( 2.5 and $5.0 \mathrm{mg} / \mathrm{kg}$, as commonly prescribed). Expected atenolol-induced hypotension was present, associated with a significantly increased heart rate and peripheral perfusion with both dosages. Through the application of WA to the LDF signal, we could detail the mechanisms of the atenolol-induced peripheral perfusion modulation: an immediate amplitude decrease of the cardiac LDF spectrum with an amplitude increase of the sympathetic component $(p<0.05)$ and the endothelial and myogenic components (non-significant). These data suggested a regulatory crosstalk between the peripheral (baroreceptors) and the microcirculatory units, which ultimately resulted in hypotension, inotropic reduction, and tachycardia. In conclusion, WA offered insight that simply could not be seen with only the perfusion curve and, thus, was an effective tool to investigate this cardiovascular mechanism of regulation.
\end{abstract}

Keywords: microcirculation; laser Doppler flowmetry; mouse flowmotion; atenolol; wavelet analysis

\section{Introduction}

In vivo models, whether animal or human, provide useful information on both physiological mechanisms and pathophysiological processes leading to disease. Vascular medicine has benefitted from the development of a wide variety of animal models with multiple approaches, such as surgical ligation-mediated ischemia [1], photochemical injury of the endothelium [2], metabolic [3], chemical [4], and hormone-induced vascular remodeling [5], which come with a combination of challenges [6] often involving genetically modified animals [7]. One of these, a murine physiological model, offers a dynamic approach to perfusion measurement continuously registered under the effect of different challengers [6,7]. The anesthetized mouse model measures perfusion in both hindlimbs with laser Doppler flowmetry (LDF) which has been applied in different experimental conditions with 
a demonstrated combination of good sensitivity and discrimination capacities [6-8]. Additionally, the model utilizes the wavelet transform analysis (WA), a scale-independent tool used to obtain a spectral decomposition of the continuous, pulsatile, raw LDF signal [9-12]. A wavelet is a small wave/oscillation that decays quickly and can adopt different shapes according to the objective of the analysis [9,13-15]. After choosing the shape of the original wavelet, also termed the "mother wavelet", a family of wavelets is obtained by stretching and shortening its length in time. Compressed wavelets are used to characterize high-frequency phenomena, while dilated wavelets are used for low-frequency phenomena. For blood peripheral perfusion variables, including cardiac, respiratory, and peripheral (myogenic, neurogenic, and endothelial) components, the Morlet mother wavelet shows good localization both in time and frequency domains, in addition to an adequate correlation between time, width, and the corresponding frequency $[9,14]$. This analytical strategy, although not common in experimental animals, was previously attempted in rats [13] and more recently in mice as part of the development of this model $[14,15]$ using human component frequencies as a reference.

In an effort to better characterize mouse microcirculatory flowmotion, the core of our study was the application of WA to LDF signals. The assessment of the embedded individual LDF components would add more depth to the signals to better understand the complex balance between components generating flowmotion. The challenger was atenolol, a well-known drug that reduces blood pressure by decreasing heart rate and contractility $[16,17]$. Its acute application has been used to assess the impact in blood pressure variability $[18,19]$. Using WA, we expected to be able to see in greater detail what happens under these conditions to the mouse flowmotion profile.

\section{Materials and Methods}

The study was previously submitted and approved by the local Animal Care and Use Committee of the Universite de Bordeaux. Six male C57/BL6 mice (16 weeks old, $24.6 \pm 1.9$ g) were used. The number of subjects was defined following our previous experience with these types of studies [6,7], taking into account the interindividual variability and also keeping in mind the guidelines from the Directive 2010/63/EU of the European Parliament on the protection of animals for scientific purposes. The animals were housed at the INSERM U1034 animal facility with controlled temperature and humidity conditions $\left(21 \pm 1{ }^{\circ} \mathrm{C}, 40-60 \%\right)$, exposed to regular $12 \mathrm{~h}$ light/ $12 \mathrm{~h}$ darkness cycles with food and water ad libitum. Animals were anesthetized with an intraperitoneal administration of a mixture of ketamine $(137.5 \mathrm{mg} / \mathrm{kg})$ and xylazine $(11 \mathrm{mg} / \mathrm{kg})$. Ten minutes after induction, animals were placed on a heated pad and allowed to stabilize for another 10 min while measurement probes were being attached. The baseline record was used as a reference, meaning that each animal served as its own control. Atenolol (Tenormine IV, $5 \mathrm{mg} / 10 \mathrm{~mL}$, AstraZeneca Ltd., Luton, UK) was then administered intraperitoneally $(5.0 \mathrm{mg} / \mathrm{kg}$ ), subdivided into two doses of $2.5 \mathrm{mg} / \mathrm{kg}$, with measurements recorded in three phases: $10 \mathrm{~min}$ baseline, $10 \mathrm{~min}$ after the first dose, and $10 \mathrm{~min}$ after the second dose. Blood pressure was monitored on the tail artery with a CODA High Throughput system (Kent Scientific, Torrington, CT, USA). Perfusion was assessed on the plantar surface of a randomly chosen hindlimb using LDF (407 probes, Perimed, Järfälla, Sweden) attached to a Periflux 5000 system (0.03 time constant, $32 \mathrm{~Hz}$ sampling rate). Heart rate was assessed by electrocardiography (ECG, lead I) using needle electrodes subcutaneously attached to the fore and hindlimbs (Power Lab/4SP, AD Instruments, Bella Vista, New South Wales, Australia). Respiratory rate was assessed by spirometry (Spirometer, AD Instruments, New South Wales, Australia). Raw LDF signals were decomposed into their spectral components (cardiac, respiratory, myogenic, sympathetic, endothelial NO-dependent, and endothelial NO-independent) with the Morlet wavelet transform (Matlab R2015, Mathworks, Natick, MA, USA). After confirmation that the samples did not follow a normal statistical distribution using the Shapiro-Wilk test, nonparametric statistics were applied for phase comparisons (Wilcoxon signed rank test) and a $p<0.05$ adopted. 


\section{Results}

Hypotension immediately followed each administration of atenolol, as expected $[16,18,19]$. Significant perfusion changes also occurred with the administration of both sequential dosages, more pronounced with the latter. The representation of the mean ( $\pm \mathrm{sd})$ LDF signal evolution along the experimental protocol is illustrated in Figure 1 and quantified in Table 1.

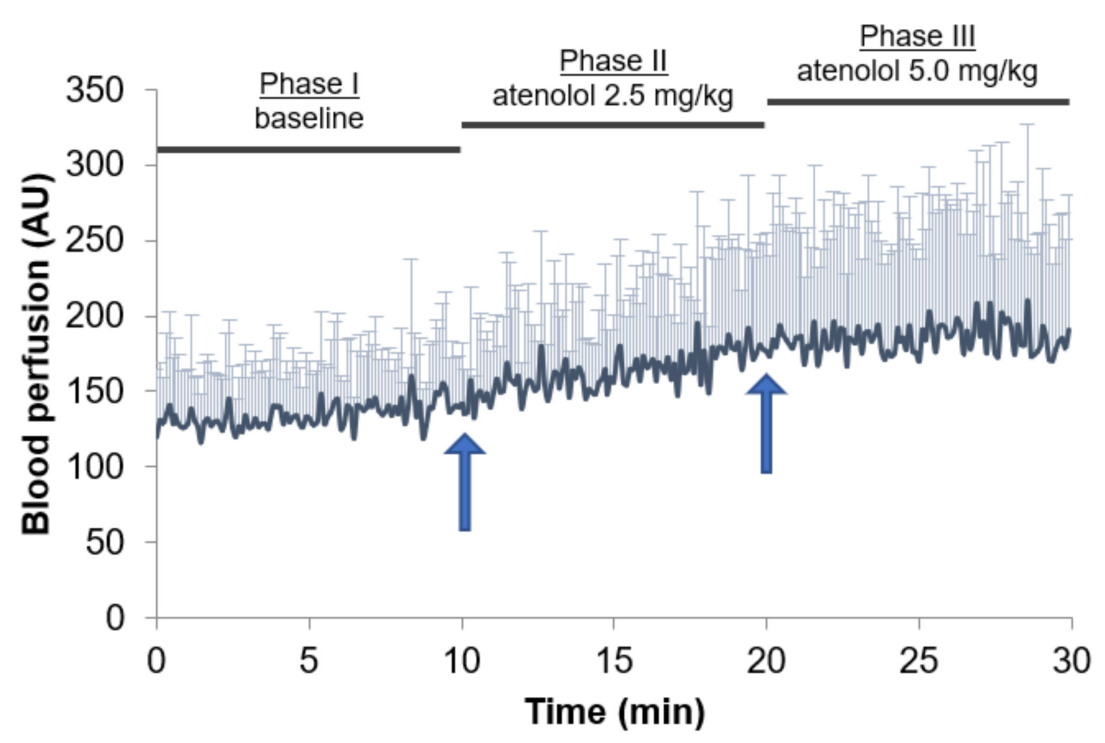

Figure 1. Mean + standard deviation (sd) of the local blood perfusion throughout the protocol $(\mathrm{N}=6)$. The arrows mark the moments of the sequential administration of two atenolol dosages $(2.5 \mathrm{mg} / \mathrm{kg}$ and $5.0 \mathrm{mg} / \mathrm{kg}$ ) (see text).

Table 1. Mean, standard deviation (sd) and percent variation $(\Delta)$ of the blood perfusion $(\mathrm{N}=6$, mean for both hindlimbs, AU—arbitrary units) in the three phases of the protocol (Phase I-baseline; Phase II-2.5 mg/kg dose \#1; Phase III— $5.0 \mathrm{mg} / \mathrm{kg}$ dose \#2). Statistical comparison ( $p$-values) versus Phase I are presented ( $\left.{ }^{*} p<0.05\right)$.

\begin{tabular}{ccccccc}
\hline Blood Perfusion (AU) & Phase I & Phase II & Phase III & $\Delta$ II-I & $\Delta$ III-I & $\Delta$ III-I \\
\hline mean & 146.4 & 172.0 & 205.3 & +17.9 & +39.1 & +17.1 \\
sd & 51.3 & 59.3 & 89.5 & +8.6 & +33.6 & +22.2 \\
$p$ & - & $0.018 *$ & $0.028^{*}$ & - & - & - \\
\hline
\end{tabular}

The WA spectrum of the LDF signals and its main component frequencies, including the lowest frequencies (endothelial), are shown in Figure 2.

With this significant perfusion increase following both atenolol dosages $(p=0.018$ for the first dose, $p=0.028$ for the second), a significant increase in heart rate ( $p=0.043$ in both cases) was also noted. WA analysis showed a decrease in the cardiac ratios, only significant with the higher dosage $(p=0.046)$ and confirmed the increase of cardiac frequency $(p=0.048$ for $2.5 \mathrm{mg} / \mathrm{kg} ; p=0.028$ for $5.0 \mathrm{mg} / \mathrm{kg}$ ) in line with the heart rate analysis (Figure 3). The respiratory rate did not change with atenolol administration (Figure 3), nor did the respiratory ratio of the LDF signal. Regarding the myogenic component, a significant decrease in the frequency of this component was noted with both doses $(p=0.028)$, but no change was noted for the ratio. Finally, the sympathetic ratio increased significantly at $2.5 \mathrm{mg} / \mathrm{kg}(p=0.028)$ and at $5 \mathrm{mg} / \mathrm{kg}(p=0.046)$. 


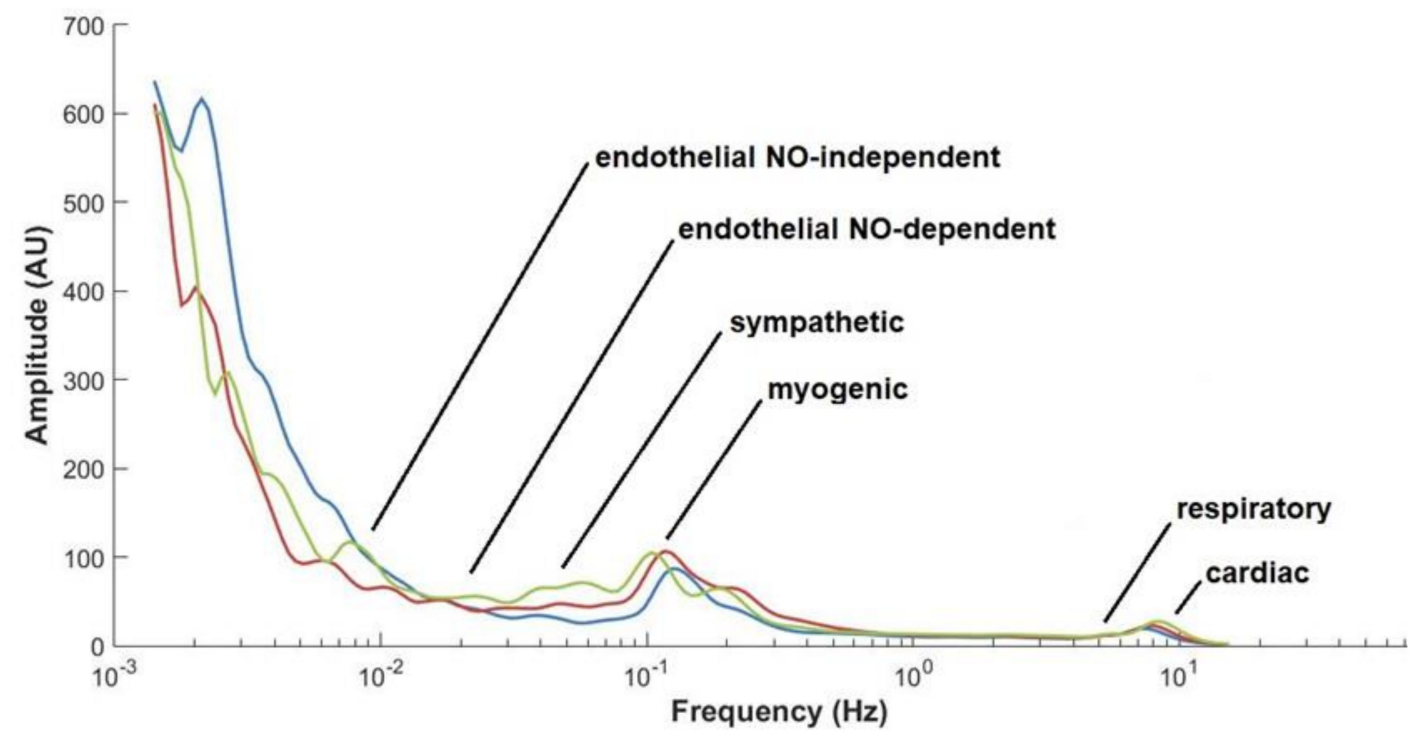

(a)

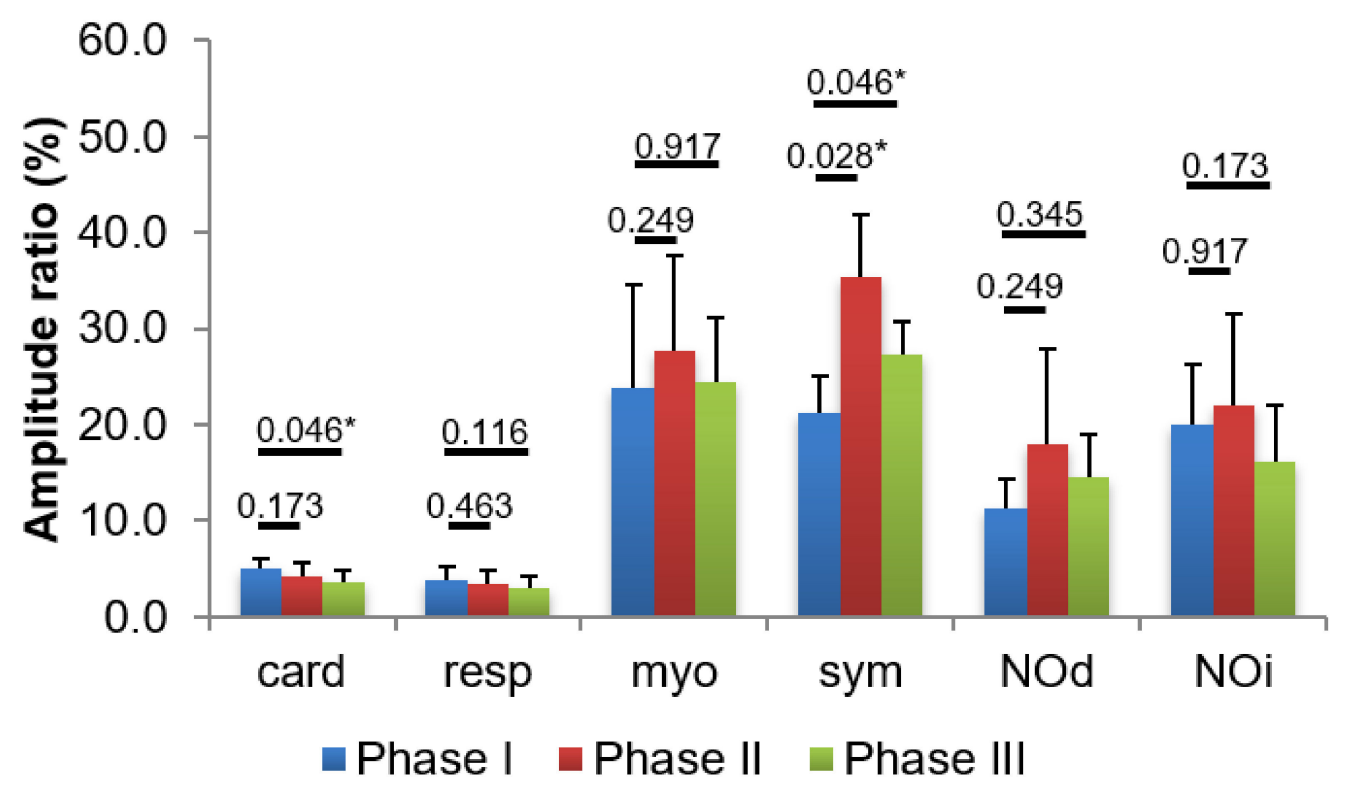

(b)

Figure 2. (a) mean spectrum of the laser Doppler flowmetry (LDF) signal for each phase of the protocol (Phase I, baseline-blue; Phase II, atenolol dose \#1—red; Phase III, atenolol dose \#2-green); (b) mean and standard deviation of the amplitude ratio for each LDF component (card—cardiac; resp—respiratory; myo-myogenic; sym-sympathetic; NOd-endothelial NO-dependent; and NOi-endothelial NO-independent). Statistical comparison ( $p$-values) versus baseline (Phase I) are shown $(* p<0.05)$. 

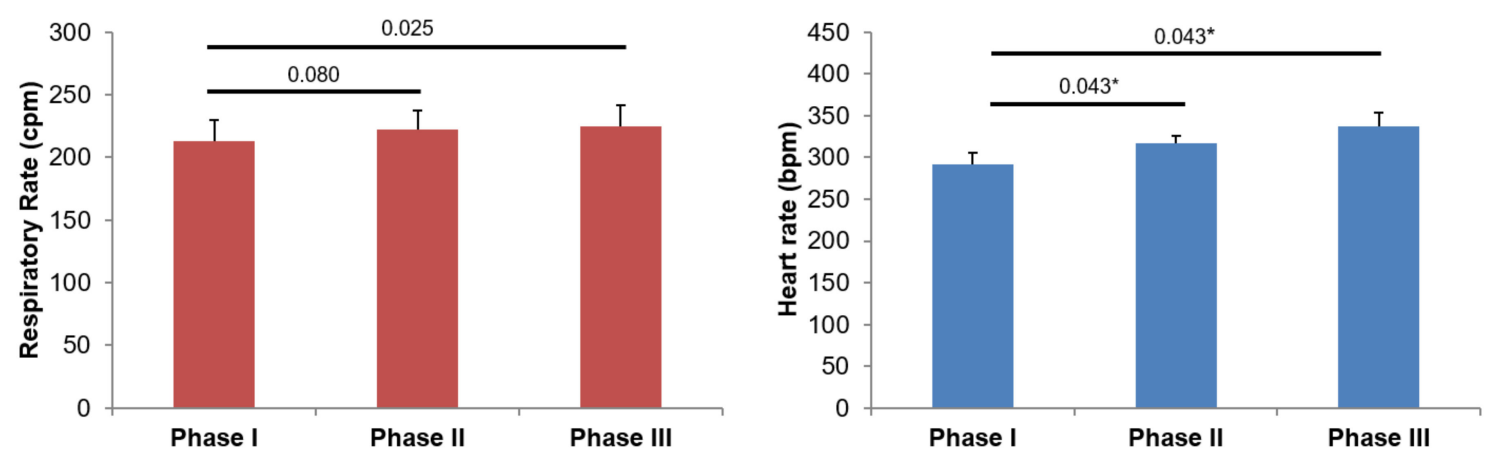

Figure 3. Mean + standard deviation of the heart rate (left) and respiratory rate (right) for the three phases of the protocol (Phase I-baseline; Phase II-atenolol dose \#1; Phase III-atenolol dose \#2). Statistical comparisons ( $p$-values) versus baseline are shown $\left({ }^{*} p<0.05\right)$.

\section{Discussion}

The selected challenger, atenolol, has been a reference in hypertensive therapy for nearly 40 years. It is often used as a standard in clinical trials looking for more effective and safe drugs for the same purposes [20]. Relevant controversies still emerge regarding its long-term impact on blood pressure variability, especially on the elderly $[17,21]$. When used in a clinical long term setting, it reduces heart rate, cardiac output, and, consequently, blood pressure, which promotes a compensatory increase in peripheral vascular resistance, reducing blood flow [16-21]. Our short-term approach results were in line with previously published data $[18,19]$. Regardless of the multiple differences in the experimental design, these acute administrations of atenolol dosages consistently decreased blood pressure with no apparent changes in the baroreflex sensitivity. Furthermore, in our case, significant increases in perfusion and heart rate were also present (Table 1 and Figure 3). No respiratory changes (respiratory rate, respiratory ratio) were observed (Figures 2 and 3) during the complete study time interval.

The proposed decomposition of LDF perfusion signals through WA brought a different look into these effects and mechanisms. Our results confirmed and complemented this notion of proximity of the mouse and rat flowmotion frequency ranges to humans. While these observations are not novel, they are important to reinforce the concept that the nature of flowmotion does not depend on the body dimension or animal species [8,13-15,22], but rather it is commonly shared with humans and other species. Anesthesia does alter these profiles, especially affecting neurogenic, myogenic, and cardiorespiratory ranges $[8,15,22]$. In previous studies using mice under ketamine-xylazine anesthesia, we found overlapping spectrum frequencies between 4.6 and $4.3 \mathrm{~Hz}$ corresponding to similar cardiac and respiratory rates ( 280 beats/minute and 240 cycles/minute, respectively) that could not be separated [8]. We described this finding as a unique cardiorespiratory band. Similar findings were reported by another research group [15] with a heart rate of $3.05 \pm 0.14 \mathrm{~Hz}$ (mean $\pm \mathrm{sd}$ ) and respiratory rate of $2.78 \pm 0.26 \mathrm{~Hz}$, suggesting that cardiac and respiratory intervals would fall under the same peak. The microcirculatory flowmotion low frequency oscillations in the mice, endothelial $(0.0095-0.021 \mathrm{~Hz})$, sympathetic $(0.021-0.052 \mathrm{~Hz})$, and myogenic $(0.052-0.145 \mathrm{~Hz})$, were suggested to be similar to those described for humans and rats [23,24]. However, some results still needed further confirmation. As ketamine has a positive chronotropic effect opposed by the negative chronotropic effect of xylazine $[15,22]$ with more effective control of the anesthesia, we were able to distinguish both cardiac and respiratory components (Figure 2a). Furthermore, we could clearly identify endothelial NO-dependent and NO-independent components. Table 2 summarizes the main reports working on these components. 
Table 2. Reported frequency ranges $(\mathrm{Hz})$ for the LDF perfusion flowmotion components in mice (see also Figure $2 b$ ) and rats, with human ranges as reference.

\begin{tabular}{|c|c|c|c|c|c|c|}
\hline \multicolumn{7}{|c|}{ Frequency Ranges (Hz) } \\
\hline & \multicolumn{2}{|c|}{ Mouse } & \multicolumn{3}{|c|}{ Rat } & \multirow{2}{*}{$\begin{array}{c}\text { Human } \\
\begin{array}{c}\text { Kvandal et al. } \\
{[11]}\end{array}\end{array}$} \\
\hline $\begin{array}{c}\text { LDF } \\
\text { Flowmotion } \\
\text { Components }\end{array}$ & $\begin{array}{l}\text { Silva et al. } \\
\text { [14] }\end{array}$ & $\begin{array}{c}\text { Astashev et al. } \\
\text { [15] }\end{array}$ & $\begin{array}{c}\text { Newman et al. } \\
\text { [13] }\end{array}$ & $\begin{array}{c}\text { Humeau et al. } \\
\text { [23] }\end{array}$ & $\begin{array}{c}\text { Geyer et al. } \\
{[24]}\end{array}$ & \\
\hline Cardiac & $4.9-8.8$ & $2.0-8.0$ & $\sim 6$ & $2.0-5.0$ & $0.4-2.0$ & $0.6-2.0$ \\
\hline Respiratory & $1.6-2.8$ & $0.2-2.0$ & $\sim 1$ & $0.74-2.0$ & $0.15-0.4$ & $0.016-0.4$ \\
\hline Myogenic & $0.09-0.26$ & $0.05-0.2$ & $\sim 0.1$ & $0.2-0.74$ & $0.05-0.15$ & $0.06-0.16$ \\
\hline Sympathetic & $0.02-0.09$ & $0.016-0.05$ & $\sim 0.05$ & $0.076-0.2$ & $0.02-0.05$ & $0.02-0.06$ \\
\hline Endothelial & & $0.005-0.016$ & $\sim 0.01$ & $0.01-0.076$ & $0.008-0.02$ & \\
\hline NO-dependent & $0.01-0.02$ & - & - & - & - & $0.0095-0.02$ \\
\hline NO-independent & $0.006-0.010$ & - & - & - & - & $0.005-0.0095$ \\
\hline
\end{tabular}

A decrease in the cardiac ratio was always present (Figure 2a,b), which was likely related to the reported hypotensive capacity of atenolol, significant with the maximal dosage. Interestingly, WA reveals a significant increase in the sympathetic ratio (Figure 2b), e.g., an increase in the sympathetic output to the peripheral vasculature after the atenolol administrations. Together with the increased heart rate, we think this results from the baroreceptor reflex evoked by the decrease in the arterial pulse pressure, even in the presence of the sympathetic-suppressing properties of ketamine-xylazine anesthesia [17]. Although non-significant, some related modifications of the myogenic and endothelial components were also observed (Figure $2 b$ ). This might also suggest that microcirculatory adaptations to perfusion involving vascular smooth muscle and endothelium also take place. With the knowledge that atenolol is not a vasoactive agent, our WA suggests the presence of more complex interactions to regulate cardiovascular homeostasis following the $\beta 1$-blockage, likely involving a combined myogenic-endothelial effect which could overcome the increased sympathetic output we detected with each dose, relative to baseline. The use of larger cohorts of subjects, alternate anesthesia approaches, and other perfusion detection technologies would be valuable to confirm and extend these findings.

\section{Conclusions}

In this study, we confirmed the mouse flowmotion mean spectrum complete with the inclusion of the lowest frequency endothelial components.

Our study also highlights the interest of this mechanistic insight offered by WA to better understand the LDF perfusion signal. WA consistently shows the progression of the perfusion adaptation process following an intervention on cardiovascular homeostasis that, in this case, results in hypotension, inotropic reduction, and tachycardia. These results seem to reveal a regulatory crosstalk between central and peripheral microcirculatory effectors, observed here in mice, as previously observed in humans. Although more data and broader research are needed, this enhanced non-invasive analysis appears to be a powerful tool for further developments in vascular medicine.

Author Contributions: L.M.R. and A.-P.G. conceived and designed research; É.R. and H.S. performed experiments and acquired data; L.M.R. and H.S. analised data and interpreted results; L.M.R. and A.-P.G. drafted, revised and approved the final version of the manuscript. All authors have read and agreed to the published version of the manuscript.

Funding: This work was supported by funds from FCT-Fundação para a Ciência e Tecnologia, I.P (Portugal) within the project UID/DTP/04567/2019 and by INSERM-U Bordeaux (France).

Conflicts of Interest: The authors declare no conflict of interest. 


\section{References}

1. Couffinhal, T.; Silver, M.; Zheng, L.P.; Kearney, M.; Witzenbichler, B.; Isner, J.M. Mouse model of angiogenesis. Am. J. Pathol. 1998, 152, 1667-1679. [PubMed]

2. Kikuchi, S.; Umemura, K.; Kondo, K.; Saniabadi, A.R.; Nakashima, M. Photochemically induced endothelial injury in the mouse as a screening model for inhibitors of vascular intimal thickening. Arter. Thromb. Vasc. Biol. 1998, 18, 1069-1078. [CrossRef] [PubMed]

3. Lee, Y.T.; Lin, H.Y.; Chan, Y.W.F.; Li, K.H.C.; To, O.T.L.; Yan, B.P.; Liu, T.; Li, G.; Wong, W.T.J.; Keung, W.; et al. Mouse models of atherosclerosis: A historical perspective and recent advances. Lipids Health Dis. 2017, 16, 12. [CrossRef] [PubMed]

4. Wang, Y.; Krishna, S.; Golledge, J. The calcium chloride-induced rodent model of abdominal aortic aneurysm. Atherosclerosis 2013, 226, 29-39. [CrossRef]

5. Cao, R.; Amand, T.; Ford, M.D.; Piomelli, U.; Funk, C. The Murine Angiotensin II-Induced Abdominal Aortic Aneurysm Model: Rupture Risk and Inflammatory Progression Patterns. Front. Pharmacol. 2010, 1, 9. [CrossRef]

6. Rodrigues, L.M.; Silva, H.; Ferreira, H.A.; Renault, M.-A.; Gadeau, A.-P. Observations on the perfusion recovery of regenerative angiogenesis in an ischemic limb model under hyperoxia. Physiol. Rep. 2018, 6, e13736. [CrossRef]

7. $\quad$ Rodrigues, L.M.; Silva, H.; Ferreira, H.A.; Gadeau, A.-P. Characterizing Vascular Dysfunction in Genetically Modified Mice through the Hyperoxia Model. Int. J. Mol. Sci. 2019, 20, 2178. [CrossRef]

8. Rodrigues, L.M.; Silva, H.; Ferreira, H.; Gadeau, A.P. This hyperoxia mouse model using the wavelet transform analysis of flowmotion signals helps to look further into microvascular dysfunction. In Proceedings of the Meeting of the French Soc, Reims, France, 15 Feburary 2019; p. 39.

9. Stefanovska, A.; Bracic, M.; Kvernmo, H.D. Wavelet analysis of oscillations in the peripheral blood circulation measured by laser Doppler technique. IEEE Trans. Biomed. Eng. 1999, 46, 1230-1239. [CrossRef]

10. Bernjak, A.; Stefanovska, A. Importance of wavelet analysis in laser Doppler flowmetry time series. In Proceedings of the 2006 International Conference of the IEEE Engineering in Medicine and Biology Society, Lyon, France, 22-26 August 2007; pp. 4064-4067.

11. Kvandal, P.; Landsverk, S.A.; Bernjak, A.; Stefanovska, A.; Kvernmo, H.D.; Kirkebøen, K.A. Low-frequency oscillations of the laser Doppler perfusion signal in human skin. Microvasc. Res. 2006, 72, 120-127. [CrossRef]

12. Sifuzzaman, M.; Islam, M.R.; Ali, M.Z. Application of Wavelet Transform and its Advantages Compared to Fourier Transform. J. Phys. Sci. 2009, 13, 121-134.

13. Newman, J.M.B.; Dwyer, R.R.; St-Pierre, P.; Richards, S.; Clark, M.G.; Rattigan, S. Decreased microvascular vasomotion and myogenic response in rat skeletal muscle in association with acute insulin resistance. J. Physiol. 2009, 587, 2579-2588. [CrossRef] [PubMed]

14. Silva, H.; Renault, M.; Ferreira, H.; Bujan, M.; Gadeau, A.; Rodrigues, L. In vivo characterization of murine and human components of (laser Doppler) flow during hyperoxia. In Proceedings of the 40th World Congress of the International Society for Biophysics and Imaging of the Skin, Lisbon, Portugal, 31 May-3 June 2016.

15. Astashev, M.E.; Serov, D.A.; Tankanag, A.T. Anesthesia effects on the low frequency blood flow oscillations in mouse skin. Ski. Res. Technol. 2018, 25, 40-46. [CrossRef] [PubMed]

16. Merli, I.P.; Levenson, J.; Filitti, V.; Simon, A. Comparative long-term vasoactive effects of atenolol and carteolol on the properties of the small and large arteries of the upper extremities in human essential hypertension. Clin. Pharmacol. Ther. 1989, 46, 686-692. [CrossRef] [PubMed]

17. Wiysonge, S.C.; Bradley, H.A.; Volmink, J.; Mayosi, B.M.; Mbewu, A.; Opie, L.H. Beta-blockers for hypertension. Cochrane Database Syst Rev. 2017, 1, CD002003. [CrossRef] [PubMed]

18. Neto, E.S.; Frutoso, J.; Somody, L.; Gharib, C.; Fortrat, J.-O. Effect of acute atenolol on short-term blood pressure variability and baroreflex sensitivity in rats. Fundam. Clin. Pharmacol. 2000, 14, 341-349. [CrossRef] [PubMed]

19. Bertera, F.M.; Del Mauro, J.S.; Lovera, V.; Chiappetta, D.; Polizio, A.H.; Taira, C.A.; Höcht, C. Acute effects of third generation $\beta$-blockers on short-term and beat-to-beat blood pressure variability in sinoaortic-denervated rats. Hypertens. Res. 2013, 36, 349-355. [CrossRef]

20. Olawi, N.; Krüger, M.; Grimm, D.; Infanger, M.; Wehland, M. Nebivolol in the treatment of arterial hypertension. Basic Clin. Pharmacol. Toxicol. 2019, 125, 189-201. [CrossRef] 
21. Vögele, A.; Hans, T.; Guiteras, A.R.; Reeves, D.; Rieckert, A.; Schlender, L.; Teichmann, A.-L.; Sönnichsen, A.; Martinez, Y.V. Effectiveness and safety of beta blockers in the management of hypertension in older adults: A systematic review to help reduce inappropriate prescribing. BMC Geriatr. 2017, 17, 224. [CrossRef]

22. Svorc, P.; Bačová, I.; Švorc, P.; Buzga, M. Autonomic Nervous System under Ketamine/xylazine and Pentobarbital Anaesthesia in a Wistar Rat Model: A Chronobiological View. Prague Med. Rep. 2013, 114, 72-80. [CrossRef]

23. Humeau, A.; Koïtka, A.; Abraham, P.; Saumet, J.-L.; L'Huillier, J.-P. Time-frequency analysis of laser Doppler flowmetry signals recorded in response to a progressive pressure applied locally on anaesthetized healthy rats. Phys. Med. Biol. 2004, 49, 843-857. [CrossRef]

24. Geyer, M.J.; Jan, Y.-K.; Brienza, D.; Boninger, M.L. Using wavelet analysis to characterize the thermoregulatory mechanisms of sacral skin blood flow. J. Rehabil. Res. Dev. 2005, 41, 797. [CrossRef] [PubMed]

(C) 2020 by the authors. Licensee MDPI, Basel, Switzerland. This article is an open access article distributed under the terms and conditions of the Creative Commons Attribution (CC BY) license (http://creativecommons.org/licenses/by/4.0/). 